\title{
ANALYSIS OF THE N PROTEIN IN FELINE CORONAVIRUS STRAINS IN ITALY
}

\author{
Mara Battilani, Ambra Foschi, Alessandra Scagliarini, Sara Ciulli, \\ Santino Prosperi, and Luigi Morganti*
}

\section{INTRODUCTION}

Feline coronaviruses (FCoVs) are responsible for an asymptomic or mild enteric infection but also cause a progressive, fatal immune-mediated disease, feline infectious peritonitis (FIP). The structural proteins of FCoVs include the spike (S), the membrane $(\mathrm{M})$, and, the most representative, the nucleocapsid protein $(\mathrm{N})$.

Coronavirus N proteins vary from 377 to 455 amino acids in length, are highly basic, have a high serine content $(7-11 \%)$, and are potential targets for phosphorylation. Antigenic studies have shown that the $\mathrm{N}$ protein is one of the immunodominant antigens in the CoV family. ${ }^{1}$ The cellular immune response against the $\mathrm{N}$ protein of some animal coronaviruses can enhance recovery from the virus infection. Immunization with a cell lysate using a recombinant baculovirus-expressing feline infectious peritonitis virus (FIPV) nucleocapsid protein was effective in preventing the progression of FIP. ${ }^{2}$

To investigate the antigenic role of the $\mathrm{N}$ protein, we carried out a computational analysis of the $\mathrm{N}$ protein of FCoV strains detected in healthy and diseased cats on the basis of the primary amino acid sequences.

\section{MATERIALS AND METHODS}

The $\mathrm{N}$ gene of FCoV strains detected in healthy and diseased cats coming from the same shelter (Table 1) was amplified by RT-PCR, and the amplicons were sequenced. Deduced amino acids sequences were aligned by ClustalW, and the alignment was visualized using the Genedoc program and Bioedit V5.06.

The PONDR program (Molecular Kinetics, Indianapolis, IN, USA, http://www pondr .com) with the VL3-BA neuronetwork feedback predictor was used to predict the order/disorder regions of the $\mathrm{N}$ proteins of FCoV strains.

*University of Bologna, Italy. 
Table 1. Details of viral strains analyzed.

\begin{tabular}{lll}
\hline & Healthy cats & \multicolumn{1}{c}{ Diseased cats } \\
\hline $352 \mathrm{C}$ & 419 brain & Dry form \\
$352 \mathrm{~N}$ & 419 kidney & Dry form \\
$352 \mathrm{~S}$ & 420 lymph nodes & Wet form \\
368 & 420 small intestine & Wet form \\
& 420 liver & Wet form \\
\hline
\end{tabular}

$\mathrm{N}$ protein phosphorylation site predictions were made with DISPHOS (DISorderenhanced PHOSphorylation predictor, http://www.ist.temple.edu/DISPHOS). Only residues with a prediction $>0.5$ are considered to be phosphorylated.

Computational analysis of the antigenic sites was carried out using the method of Kolaskar and Tongaonkar. ${ }^{3}$

Prediction of the immunodominant helper T-lymphocyte antigenic sites from amino acid sequence data, was carried out using the AMPHI algorithm; the identification of the antigenic sites that interact with mouse MHC II haplotype d was carried out using the SETTE algorithm. ${ }^{4}$ Both methods were available in the Protean program of the DNASTAR multiple program package (Lasergene Inc., USA).

\section{RESULTS AND DISCUSSION}

The predicted amino acid sequences of the $\mathrm{N}$ gene are formed by 377 residues (the virulent strains 419 and 420) or 376 residues (the avirulent strains $352 \mathrm{~N}, \mathrm{C}, \mathrm{S}$ and 368 ) as a consequence of the deletion of residue 205.

The alignment of the $\mathrm{N}$ sequences of virulent and avirulent strains showed that the major part of the mutations are located between aa 180-230 (Fig. 1). Analysis by the PONDR program suggested that a significant part of the $\mathrm{N}$ protein is disordered, but the virulent strains seem to be slightly more disordered in the region between aa 130-240, where the major numbers of mutations are located (Fig. 2).

Recent studies have found that unfolded protein or uncostructed protein regions are involved in molecular interactions such as receptor/ligand, protein/RNA, protein/protein. Disordered protein regions play an important role in cell signaling pathways.

To predict the phosphorylation sites in our sequences, we used the Web tool DISPHOS, which uses disorder information to detect phosphorylation sites. The virulent strains showed a major number of phosporylated serines with respect to avirulent strains, and all phosphorylation sites fall in the disordered regions.

Phosphorylated $\mathrm{N}$ protein bound to viral RNA with a higher binding affinity than non-viral RNA, ${ }^{5}$ suggesting that phosphorylation of the $\mathrm{N}$ protein determined the recognition of viral RNA. Because the virulent strains showed more phosphorylation sites, we speculated that these viruses are more efficacious in assembly and packaging during viral replication. 


\begin{tabular}{|c|c|c|c|}
\hline & 200 & 220 & 240 \\
\hline & \multicolumn{3}{|c|}{ QQSNNQNTNVEDTIVAVLQKLGVTDKQRSRSKSRERSSSNSRDTTPKNANKHTWKKTAGKGEVT } \\
\hline 420_liver & \multicolumn{3}{|c|}{ 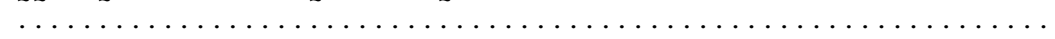 } \\
\hline 420_lymph & \multicolumn{3}{|c|}{ 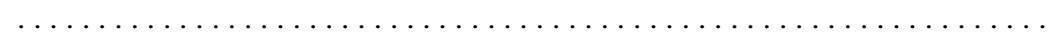 } \\
\hline 420_int. & \multicolumn{3}{|c|}{ 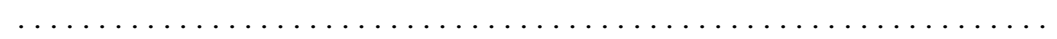 } \\
\hline 419_kidn. & \multicolumn{3}{|c|}{ 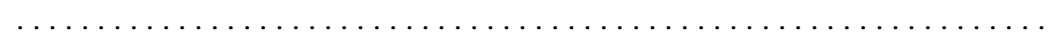 } \\
\hline 419_brain & \multicolumn{3}{|c|}{ 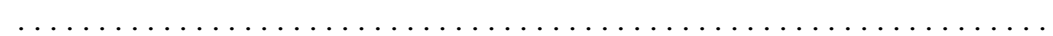 } \\
\hline 3 & \multicolumn{3}{|c|}{ 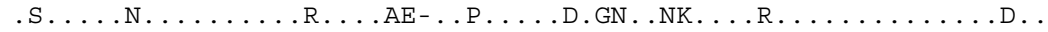 } \\
\hline 368 & \multicolumn{3}{|c|}{$. \mathrm{S} \ldots \mathrm{N} \ldots \ldots \mathrm{R}, \ldots \mathrm{AE}-\ldots \mathrm{P} \ldots \mathrm{R} \cdot \mathrm{GN}, \mathrm{NK} \ldots \mathrm{R} \ldots \ldots \ldots \mathrm{D}}$, \\
\hline
\end{tabular}

Figure 1. Alignment of predicted peptide sequence from the $\mathrm{N}$ gene. Nucleotide sequences from $\mathrm{N}$ PCR product were translated into amino acid sequences and aligned using ClustalW. Residues identical to the consensus are indicated by dots and gaps are indicated as dashes.

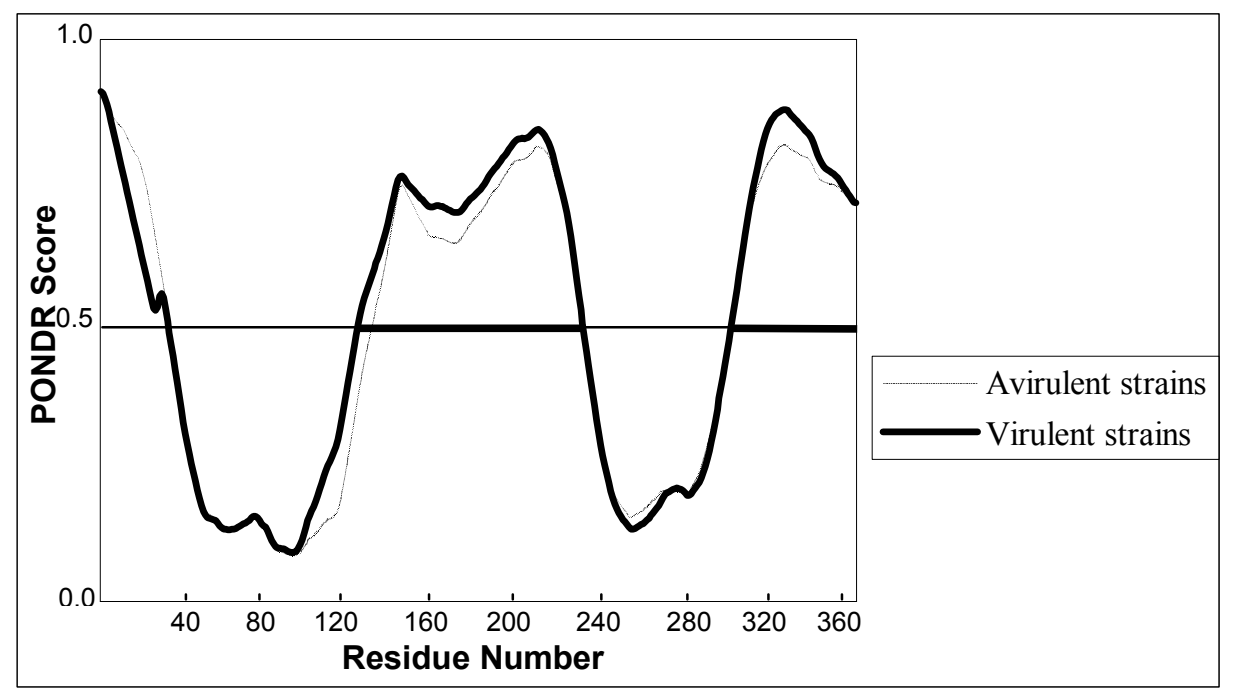

Figure 2. Order/disorder analysis of FCoVs N protein by the VL3-BA predictor of the PONDR program.

Antigenic analysis of the $\mathrm{N}$ protein was carried out by applying several methods. Our analysis demonstrated a substantial difference between virulent and avirulent strains in the mapping of antigenic sites. Using the method of Kolaskar and Tongaonkar, we predicted 13 antigenic sequences in the $\mathrm{N}$ protein of avirulent strains and 12 immunodominant sites in the virulent strains.

Antigenic analysis of $\mathrm{N}$ proteins carried out using SETTE and AMPHI algorithms showed different putative epitopes recognized by helper $\mathrm{T}$ cells and peptide antigenic sites confirming a possible involvement of the nucleocaspid protein in the protective immune response. All avirulent strains from healthy cats showed two additional motifs for IAd haplotypes detected using the Sette algorithm which are not present in other strains (aa 176-181; aa 261-266). On the basis of these results, we feel that virulent 
strains could elude immune surveillance, removing the immunodominant motifs from the $\mathrm{N}$ protein sequences.

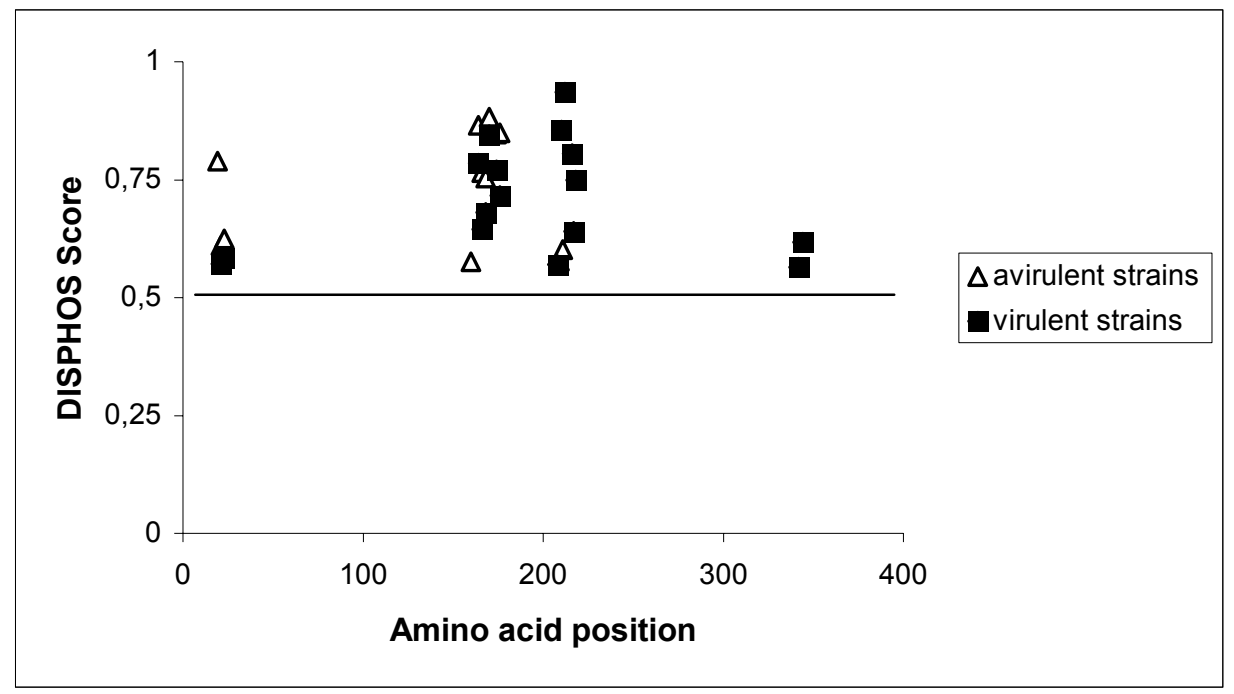

Figure 3. Prediction of phosphorylation sites.

It is interesting to compare the antigenic sites on $\mathrm{N}$ proteins with ordered/disordered regions in the amino acid sequence. The additional immunodominant site identified in the avirulent strains using the method of Kolaskar and Tongaonkar (sequence 12 TRKSCSK aa 336-342) lies in the disordered region at the border between the ordered and the disordered regions. The additional antigenic site (aa 176-181), detected using the Sette alghoritm, also falls in the disordered regions. As disordered regions are involved in molecular interaction, having the property of high specificity with modest binding affinity, the combined approach ordered/disordered regions and computational analysis of antigenic sites could be useful in the prediction of the antigenicity of the proteins.

\section{REFERENCES}

1. Lecomte, J., Cainelli-Gebara, V., Mercier, V., Mansour, S., Talbot, P. J., Lussier, G., and Oth, D., 1987, Protection from mouse hepatitis virus type 3-induced acute disease by an anti-nucleoprotein monoclonal antibody, Arch. Virol. 97:123.

2. Hohdatsu, T., Yamato, H., Ohkawa, T., Kaneko, M., Motokawa, K., Kusuhara, H., Kaneshima, T., Arai, S., and Koyama, H., 2003, Vaccine efficacy of a cell lysate with recombinant baculovirus-expressed feline infectious peritonitis (FIP) virus nucleocapsid protein against progression of FIP. Vet. Microbiol. 97:31.

3. Kolaskar, A. S., and Tongaonkar, P. C., 1990, A semi-empirical method for prediction of antigenic determinants on protein antigens, FEBS Lett. 276:172.

4. Sette, A., Buus, S., Appella, E., Smith, J. A., Chesnut, R., Miles, C., Colon, S. M., and Grey, H. M., 1989 , Prediction of major histocompatibility complex binding regions of protein antigens by sequence pattern analysis, Proc. Natl. Acad. Sci. USA 86:3296.

5. Chen, H., Gill, A., Dove, B. K., Emmett, S. R., Kemp, C. F., Ritchie, M. A., Dee, M., and Hiscox, J. A., 2005, Mass spectroscopic characterization of the coronavirus infectious bronchitis virus nucleoprotein and elucidation of the role of phosphorylation in RNA binding by using surface plasmon resonance, J. Virol. 79: 1164. 